\title{
Uma Abordagem para Geração Automática de Conteúdo Personalizado Através da Recomendação Estocástica de Objetos de Aprendizagem no Processo de Ensino em Sistemas Adaptativos e Inteligentes para Educação
}

\author{
Fabiano A. Dorça ${ }^{1}$, Daydson H. Silva ${ }^{1}$, Luciano V. Lima ${ }^{2}$, Márcia A. Fernandes ${ }^{1}$, Carlos R. Lopes $^{1}$ \\ ${ }^{1}$ Faculdade de Computação (FACOM) - Universidade Federal de Uberlândia (UFU) \\ ${ }^{2}$ Faculdade de Engenharia Elétrica (FEELT) - Universidade Federal de Uberlândia (UFU) \\ \{fabiano, marcia,crlopes\}@facom.ufu.br, vieira@ufu.br, daydson.silva@hotmail.com
}

\begin{abstract}
Resumo. A personalização automática do processo de ensino baseada em estilos de aprendizagem é um aspecto fundamental em sistemas adaptativos e inteligentes para educação, sendo esta, atualmente, uma área sob intensa investigação. Neste contexto, este trabalho apresenta um modelo baseado em regras de produção e probabilidades para geração automática de conteúdo personalizado através da recomendação estocástica de objetos de aprendizagem no processo de ensino nestes sistemas. Experimentos realizados com o modelo proposto mostraram resultados promissores. Os testes realizados apresentaram a recomendação, ordenação e destaque dos OAs de maneira eficaz, levando em consideração os estilos de aprendizagem dos estudantes.
\end{abstract}

\begin{abstract}
Automatic customization of teaching process based on learning styles is a key aspect in intelligent and adaptive educational systems, which is currently under intense investigation. In this context, this paper presents a model based on production rules and probabilities for automatic generation of personalized content through the stochastic recommendation of learning objects during the teaching process in these systems. Experiments conducted with the proposed model showed promising results. The tests showed effective recommendation of learning objects, taking into account students learning styles.
\end{abstract}

Palavras-chave - sistemas adaptativos e inteligentes para educação, modelagem do estudante, estilos de aprendizagem, recomendação de objetos de aprendizagem.

Agradecimento - Este trabalho contou com o apoio da Fundação de Amparo à Pesquisa do Estado de Minas Gerais (FAPEMIG).

\section{Introdução}

Sistemas adaptativos e inteligentes para educação (SAIE) devem ser capazes de adaptar o conteúdo e o modo de apresentação de acordo com o perfil e preferências específicas de um estudante, com objetivo de se tornar o processo de ensino mais eficiente [Graf e Kinshuk 2010].

O uso de técnicas de hipermídia adaptativa para promover personalização e facilitar o aprendizado passou a ser percebido [Brusilovsky et al. 1998, Graf e Kinshuk 2010]. 
Nesse contexto, surgem as ideias de adaptação de um conteúdo a ser ensinado às características específicas de quem o aprenderá [Bittencourt e Costa 2011, Nat et al. 2010].

Um grande número de estudos atesta que a aprendizagem é facilitada se as estratégias pedagógicas estiverem de acordo com os estilos de aprendizagem (EA) do estudante, e afirmam que utilizar material e atividades instrucionais que vão ao encontro dos EA, torna o processo de aprendizagem mais efetivo e a performance do estudante é consideravelmente melhorada, conforme apresentado em diversos trabalhos [Haider et al. 2010, Kinshuk et al. 2009, Felder e Silverman 1988].

Neste contexto, este trabalho apresenta uma nova abordagem para geração automática de conteúdo de hipermídia adaptado às preferências de EA dos estudantes. Para isto, propõe-se a utilização de uma abordagem para modelagem automática e dinâmica de EA de estudantes baseada em aprendizagem por reforço, inicialmente apresentada em [Dorça et al. 2013a]. Além disto, adapta-se e aplica-se o método proposto por [Graf e Kinshuk 2010], chamado mecanismo flexível, para recomendação de objetos de aprendizagem (OA) mais relevantes aos EA do estudante. Este último utiliza técnicas de adaptação de suporte à navegação para recomendar conteúdo mais relevantes ao perfil do estudante, adaptando a sequência dos links, quantidade de objetos, anotando, escondendo ou destacando links.

Como resultado, obteve-se uma abordagem que permite aos tutores adicionar adaptatividade aos seus cursos existentes, utilizando uma estrutura flexível de curso. A próxima seção apresenta alguns trabalhos relacionados. A seção 3 apresenta a abordagem proposta. A seção 4 apresenta alguns experimentos e resultados obtidos. A seção 5 apresenta conclusões e trabalhos futuros.

\section{Abordagem Proposta}

Neste ponto, é importante apresentar alguns detalhes das abordagens propostas por [Graf e Kinshuk 2010] e [Dorça et al. 2013a] para que se entenda o modelo proposto neste trabalho. Um ponto importante em comum, e que possibilita propor um modelo que as agregue, é que ambas são baseadas no modelo proposto por Felder e Silverman (1988) para EA [Felder e Silverman 1988].

A aplicação do método flexível, que se dá em dois estágios, presume a existência de uma lição e um modelo de estudante. O primeiro estágio é a anotação, onde os conteúdos, cujos tipos têm afinidade com o estilo do aluno a ser atendido são marcados como recomendados, da mesma forma que os objetos considerados essenciais: comentário inicial, conteúdo, conclusão e avaliação. Como será apresentado a seguir, o método proposto por [Graf e Kinshuk 2010] considera 12 diferentes tipos de objetos de aprendizagem.

Para a compreensão do segundo estágio do método, é necessário ter em mente a estrutura da página a ser montada, que está representada na Figura 1. Na referida estrutura, o objeto Conteúdo sempre existirá por ser a única exigência para que o método seja aplicado. Os objetos Comentário e Avaliação, caso existam, serão o primeiro e o último da lição, respectivamente. O segundo estágio é a ordenação, que determinará a posição dos demais conteúdos dentro da página. [Graf e Kinshuk 2010] cita uma série associações entre os EAs e os tipos de conteúdos considerados no método. Tais associações, bem como 


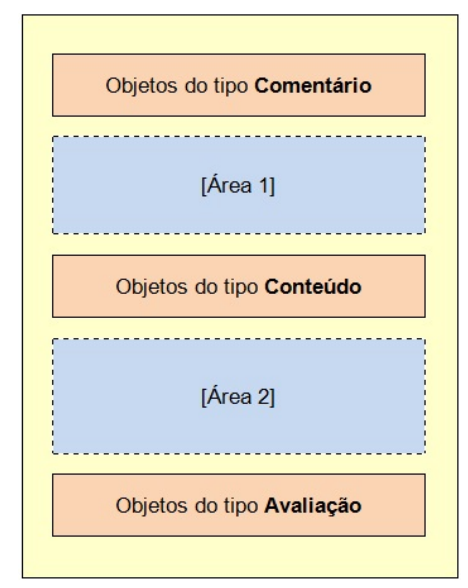

Figura 1. Estrutura da página que representa uma lição.

a recomendação de aparecerem na Área 1 (A1) ou na Área 2 (A2) estão representadas na Tabela 1. Para ambas as áreas, os conteúdos são incluídos em ordem decrescente de afinidade (peso), de acordo com o modelo do estudante (ME), como será explicado posteriormente. De acordo com [Graf e Kinshuk 2010], na área 1 é recomendada a utilização de, no máximo, dois tipos de objetos, para não sobrecarregar o aprendiz com atividades antes do contato com o real conteúdo da lição.

Tabela 1. Adequação e posicionamento dos conteúdos às áreas 1 ou 2 , conforme perfil do estudante.

\begin{tabular}{|l|c|c|c|c|c|c|c|c|}
\hline \multirow{2}{*}{ Tipos de Objetos } & \multicolumn{9}{|c|}{ PERFIL DO ESTUDANTE } \\
\cline { 2 - 9 } & Processamento & \multicolumn{2}{c|}{ Percepção } & \multicolumn{2}{c|}{ Entrada } & \multicolumn{2}{c|}{ Organização } \\
\cline { 2 - 9 } & Ativo & Reflexivo & Sensitivo & Intuitivo & Visual & Verbal & Sequencial & Global \\
\hline Questionário de Reflexão & - & A2 & - & A2 & - & - & - & - \\
\hline Teste de auto-avaliação & A1 & - & A2 & - & - & - & - & - \\
\hline Fórum de discussão & A2 & - & - & - & - & A2 & - & - \\
\hline Leitura Adicional & - & A2 & - & A2 & - & A2 & - & - \\
\hline Animação & A1 & - & A1 & - & A1 & - & - & - \\
\hline Exercício & A1 & - & A2 & A1 & - & - & - & - \\
\hline Exemplo & - & A2 & A1 & - & - & - & - & A2 \\
\hline Aplicações na vida real & - & - & A1 & - & - & - & - & A2 \\
\hline
\end{tabular}

Objetos do tipo conclusão figuram sempre no início ou no fim da área 2, a depender do perfil do estudante, como visto na Tabela 2, enquanto as avaliações, invariavelmente, encerram a estrutura da página.

Tabela 2. Posicionamento do objeto Conclusão na Área 2, conforme perfil do estudante.

\begin{tabular}{|l|c|c|c|c|c|c|c|c|}
\hline \multirow{3}{*}{ Tipo de Objeto } & \multicolumn{9}{|c|}{ PERFIL DO ESTUDANTE } \\
\cline { 2 - 9 } & Processamento & \multicolumn{2}{|c|}{ Percepção } & \multicolumn{2}{c|}{ Entrada } & \multicolumn{2}{c|}{ Organização } \\
\cline { 2 - 8 } & Ativo & Reflexivo & Sensitivo & Intuitivo & Visual & Verbal & Sequencial & Global \\
\hline Conclusão & Fim & Início & Fim & Fim & - & Fim & Fim & Início \\
\hline
\end{tabular}

$\mathrm{Na}$ abordagem proposta neste trabalho, as preferências dos estudantes são armazenadas através de distribuições de probabilidades no ME, indicando as probabilidades de preferência por cada EA dentro de cada uma das quatro dimensões do modelo de Felder-Silverman, chamados aqui de EA probabilísticos $\left(E A_{p}\right)$. Então, tem-se um ME 
probabilístico em que EA são tratados pelo sistema como probabilidades, e não como certezas. A Tabela 3 apresenta um exemplo de $E A_{p}$ no ME, representando um estudante que provavelmente é Reflexivo, Intuitivo, Visual e Sequencial.

Tabela 3. Exemplo de Inicialização dos $E A_{p}$

\begin{tabular}{|c|c|c|c|c|c|c|c|}
\hline \multicolumn{8}{|c|}{ EA Probabilísticos $\left(E A_{p}\right)$} \\
\hline \multicolumn{2}{|c|}{ Processamento } & \multicolumn{2}{c|}{ Percepção } & \multicolumn{2}{c|}{ Entrada } & \multicolumn{2}{c|}{ Organização } \\
\hline Ativo & Reflexivo & Sensitivo & Intuitivo & Visual & Verbal & Sequencial & Global \\
\hline 0,35 & 0,65 & 0,17 & 0,83 & 0,89 & 0,11 & 0,84 & 0,16 \\
\hline
\end{tabular}

Caso seja utilizado um questionário inicial para auto-avaliação de EA compatível com o modelo de Felder-Silverman, como o Index of Learning Styles Questionnaire (ILSQ) [Van Zwanenberg et al. 2000], o ME pode ser inicializado através dos dados obtidos pelo questionário, fazendo-se a conversão considerando-se a proporcionalidade das respostas pontuadas para cada EA, dividindo-se a quantidade de respostas favoráveis a um EA pelo total de respostas dentro da dimensão (11 para cada dimensão, totalizando 44 questões no ILSQ). Caso nenhum questionário para a identificação inicial de EAs dos estudantes seja utilizado, todos os $E A_{p}$ no $\mathrm{ME}$ são inicializados em 0,50 (preferência indefinida).

Desta forma, tem-se um ME probabilístico em que EA são tratados pelo sistema como probabilidades, e não como certezas. Os processos de modelagem automática e dinâmica dos $E A_{p}$ do estudante, e de seleção das estratégias de ensino são baseados na seleção estocástica de uma combinação de EA (CEA) que defina uma ação pedagógica apropriada a ser tomada em determinado momento.

A cada seção de aprendizagem, uma CEA é selecionada estocasticamente, de acordo com as probabilidades dadas pelos $E A_{p}$. Uma CEA é formada por 4 variáveis probabilísticas, definidas pelas 4 dimensões do modelo de Felder-Silverman, como apresentado em 2.1. Cada variável possui 2 valores possíveis, e cada valor representa um EA cujas possibilidades de assumir um valor ou outro é dado por sua probabilidade de preferência pelo estudante, dada por $E A_{p}$. Desta forma, é proposta uma abordagem regida por um processo estocástico baseado nas 4 dimensões de EA do modelo de FelderSilverman. Neste contexto, uma grande vantagem desta abordagem é considerar estocasticamente, durante o processo de aprendizagem, todas as CEAs de acordo com os $E A_{p}$, que podem estar inconsistentes ou variar ao longo do tempo em decorrência de algum aspecto, conforme discutido anteriormente.

Definição 2.1 Combinação de estilos de aprendizagem (CEA)

$C E A=(a, b, c, d \mid a \in D 1, b \in D 2, c \in D 3, d \in D 4)$

considerando:

$D 1=\{\operatorname{Ativo}(A), \operatorname{Reflexivo}(R)\}$;

$D 2=\{\operatorname{Sensitivo}(S)$, Intuitivo $(I)\}$

$D 3=\{\operatorname{Visual}(V i), \operatorname{Verbal}(V e)\}$

$D 4=\{\operatorname{Sequencial}(\operatorname{Seq}), \operatorname{Global}(G)\}$.

Considerando os $E A_{p}$, a probabilidade $P$ de pertinência do estudante a uma categoria pedagógica é dada por (1), que é a mesma de uma CEA ser selecionada durante uma seção de aprendizagem, que é igual à probabilidade de preferência do estudante pela 
CEA. A probabilidade $P$ é calculada através de (1), em que $a, b, c, d$ é dado pela Definição 2.1 .

$$
P(a, b, c, d)=P r_{a} \times P r_{b} \times P r_{c} \times P r_{d}
$$

Desta forma, de acordo com (1), a probabilidade de preferência do estudante pela CEA (A,S,Vi,Seq), considerando-se o ME dado pela Tabela 3, é dada por:

$$
\begin{gathered}
P(A, S, V i, S e q)=P r_{A} \times P r_{S} \times P r_{V i} \times P r_{S e q} \\
P(A, S, V i, S e q)=0,35 \times 0,17 \times 0,89 \times 0,84=0.045
\end{gathered}
$$

Então, aplicando-se (1) às CEAs dadas por 2.1, tem-se a distribuição de probabi-

\begin{tabular}{|c|c|}
\hline CEA & Probabilidades \\
\hline $\mathrm{P}(\mathrm{A}, \mathrm{S}, \mathrm{Vi}, \mathrm{Seq})$ & $0,35 \times 0,17 \times 0,89 \times 0,84=0,045$ \\
\hline $\mathrm{P}(\mathrm{A}, \mathrm{S}, \mathrm{Vi}, \mathrm{G})$ & $0,35 \times 0,17 \times 0,89 \times 0,16=0,008$ \\
\hline $\mathrm{P}(\mathrm{R}, \mathrm{S}, \mathrm{Vi}, \mathrm{Seq})$ & $0,65 \times 0,17 \times 0,89 \times 0,84=0,083$ \\
\hline $\mathrm{P}(\mathrm{R}, \mathrm{S}, \mathrm{Vi}, \mathrm{G})$ & $0,65 \times 0,17 \times 0,89 \times 0,16=0,016$ \\
\hline $\mathrm{P}(\mathrm{A}, \mathrm{S}, \mathrm{Ve}, \mathrm{Seq})$ & $0,35 \times 0,17 \times 0,11 \times 0,84=0,005$ \\
\hline $\mathrm{P}(\mathrm{A}, \mathrm{S}, \mathrm{Ve}, \mathrm{G})$ & $0,35 \times 0,17 \times 0,11 \times 0,16=0,002$ \\
\hline $\mathrm{P}(\mathrm{R}, \mathrm{S}, \mathrm{Ve}, \mathrm{Seq})$ & $0,65 \times 0,17 \times 0,11 \times 0,84=0,010$ \\
\hline $\mathrm{P}(\mathrm{R}, \mathrm{S}, \mathrm{Ve}, \mathrm{G})$ & $0,65 \times 0,17 \times 0,11 \times 0,16=0,003$ \\
\hline $\mathrm{P}(\mathrm{A}, \mathrm{I}, \mathrm{Vi}, \mathrm{Seq})$ & $0,35 \times 0,83 \times 0,89 \times 0,84=0,217$ \\
\hline $\mathrm{P}(\mathrm{A}, \mathrm{I}, \mathrm{Vi}, \mathrm{G})$ & $0,35 \times 0,83 \times 0,89 \times 0,16=0,043$ \\
\hline $\mathrm{P}(\mathrm{R}, \mathrm{I}, \mathrm{Vi}, \mathrm{Seq})$ & $0,65 \times 0,83 \times 0,89 \times 0,84=0,403$ \\
\hline $\mathrm{P}(\mathrm{R}, \mathrm{I}, \mathrm{Vi}, \mathrm{G})$ & $0,65 \times 0,83 \times 0,89 \times 0,16=0,076$ \\
\hline $\mathrm{P}(\mathrm{A}, \mathrm{I}, \mathrm{Ve}, \mathrm{Seq})$ & $0,35 \times 0,83 \times 0,11 \times 0,84=0,026$ \\
\hline $\mathrm{P}(\mathrm{A}, \mathrm{I}, \mathrm{Ve}, \mathrm{G})$ & $0,35 \times 0,83 \times 0,11 \times 0,16=0,005$ \\
\hline P(R,I,Ve,Seq) & $0,65 \times 0,83 \times 0,11 \times 0,84=0,049$ \\
\hline $\mathrm{P}(\mathrm{R}, \mathrm{I}, \mathrm{Ve}, \mathrm{G})$ & $0,65 \times 0,83 \times 0,11 \times 0,16=0,009$ \\
\hline Soma das probabilidades & 1,000 \\
\hline
\end{tabular}
lidades apresentadas na Tabela 4, considerando-se o ME dado pela Tabela 3.

Tabela 4. Distribuição de Probabilidades das CEAs

Desta forma, uma vantagem desta abordagem é considerar estocasticamente todas as CEAs de acordo com os $E A_{p}$ do estudante, que pode ser inconsistente, ou seja, pode não representar a preferência real do estudante. Esta característica permite a descoberta eficiente e eficaz dos EAs do estudante. Então, esta abordagem é intrinsecamente capaz de lidar com a incerteza no processo de modelagem de EA. A incerteza aparece devido à diversidade de fatores que exercem influência sobre o processo de aprendizagem e desempenho do estudante, como afirmam [Haider et al. 2010, Kinshuk et al. 2009, Felder e Silverman 1988].

Um processo baseado em aprendizagem por reforço é responsável por atualizar constantemente os $E A_{p}$ do estudante, que evolui de forma iterativa e incremental ao longo do processo de aprendizagem. Desta forma, as preferências de EA do estudante podem ser automaticamente detectadas, quando não se tem informações sobre elas, ou mesmo corrigidas, quando estiverem inconsistentes. Este processo é descrito em detalhes em [Dorça et al. 2013a], e atualiza os $E A_{p}$ do estudante sempre que um desempenho insatisfatório é detectado. 
Para a obtenção da sequência de conteúdos que melhor se adapta às preferências do estudante, é atribuído um peso a cada objeto disponível para determinada lição, o qual resulta da soma dos valores dos $E A_{p}$ cujos EAs pertençam à CEA selecionada durante determinada seção de aprendizagem. Como exemplo, se um objeto do tipo Leitura Adicional está disponível em uma lição e a CEA gerada possui os EA Reflexivo com $E A_{p}=0,8$ e Intuitivo com $E A_{p}=0,7$, então, o peso do objeto na lição é de 1,5 . O resultado final do processo de adaptatividade é, inicialmente, uma página em formato HTML que contém links para os diversos conteúdos recomendados, apresentados na ordem decrescente de pesos. Este resultado é gerado por um modelo que agrega os principais elementos das abordagens propostas por [Dorça et al. 2012] e [Graf e Kinshuk 2010], e apresentadas nesta seção. O modelo é apresentado pelo algoritmo 2.1.

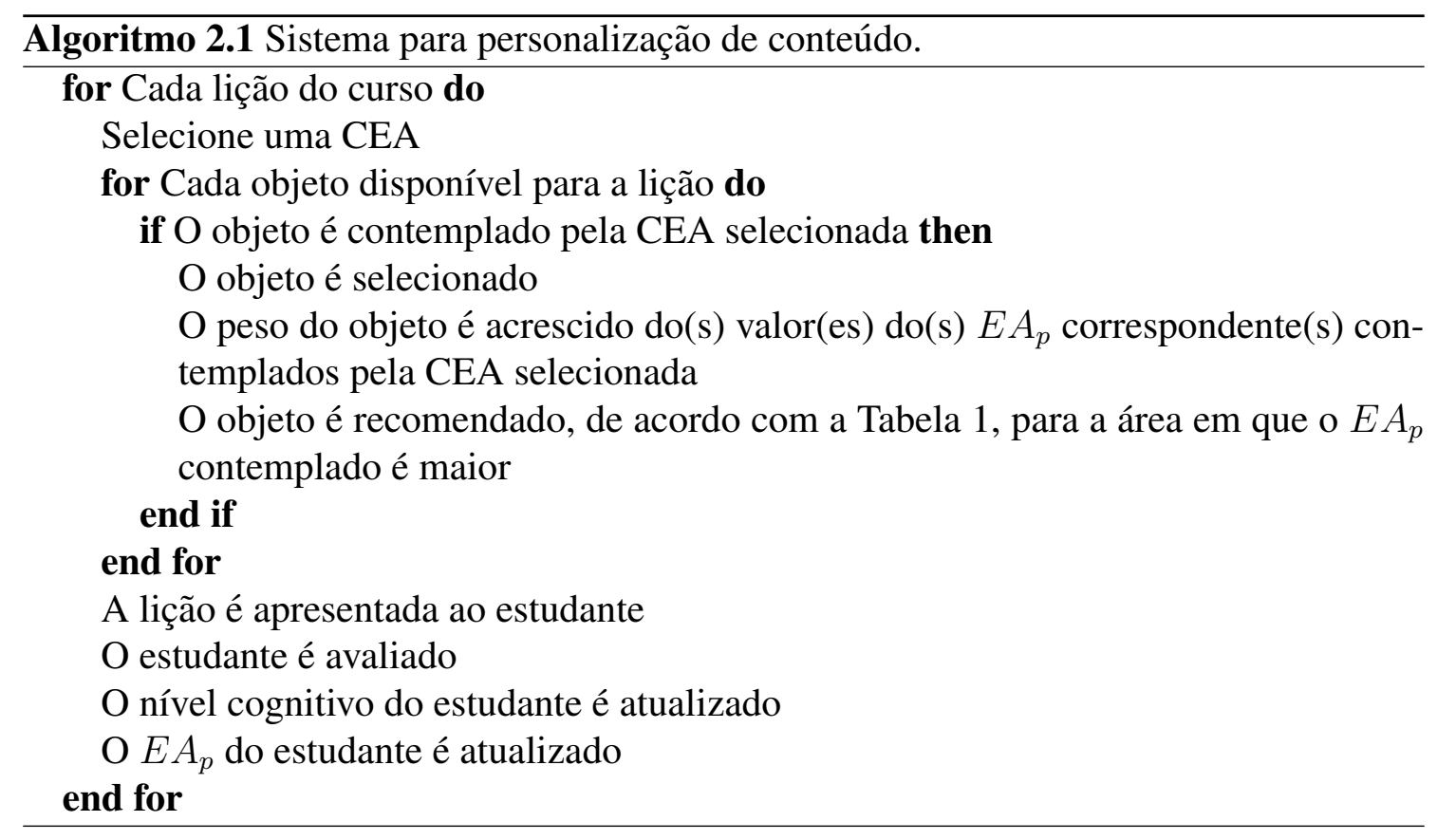

É possível notar que se um conteúdo é recomendado para diferentes áreas, dependendo do EA, então, ele será posicionado na área correspondente ao maior $E A_{p}$. Por exemplo, se um conteúdo do tipo Teste de auto-avaliação é contemplado pela CEA selecionada e recomendado para a Área 1 (EA Ativo) com peso $E A_{p}=0,35$, e para a Área 2 (EA Sensitivo) com peso $E A_{p}=0,17$, então, este objeto será posicionado na Área 1, com peso 0,52. Além disto, para posicionamento de conteúdos do tipo Conclusão, baseando-se na Tabela 2, utiliza-se o algoritmo 2.2. A próxima seção apresenta um experimento com a abordagem proposta nesta seção. Os resultados foram obtidos através de um modelo de simulação computacional do desempenho do estudante, apresentado em [Dorça et al. 2013b].

\section{Experimentação do Modelo e Resultados Obtidos}

O desenvolvimento de SAIE é uma tarefa árdua, e o resultado final é difícil de avaliar [Virvou et al. 2003]. Diante disto, técnicas de simulação computacional são essenciais e podem contribuir muito na experimentação de novas abordagens no processo de ensino, já que testar este tipo de sistema requer quantia considerável de recursos humanos (tutores e 


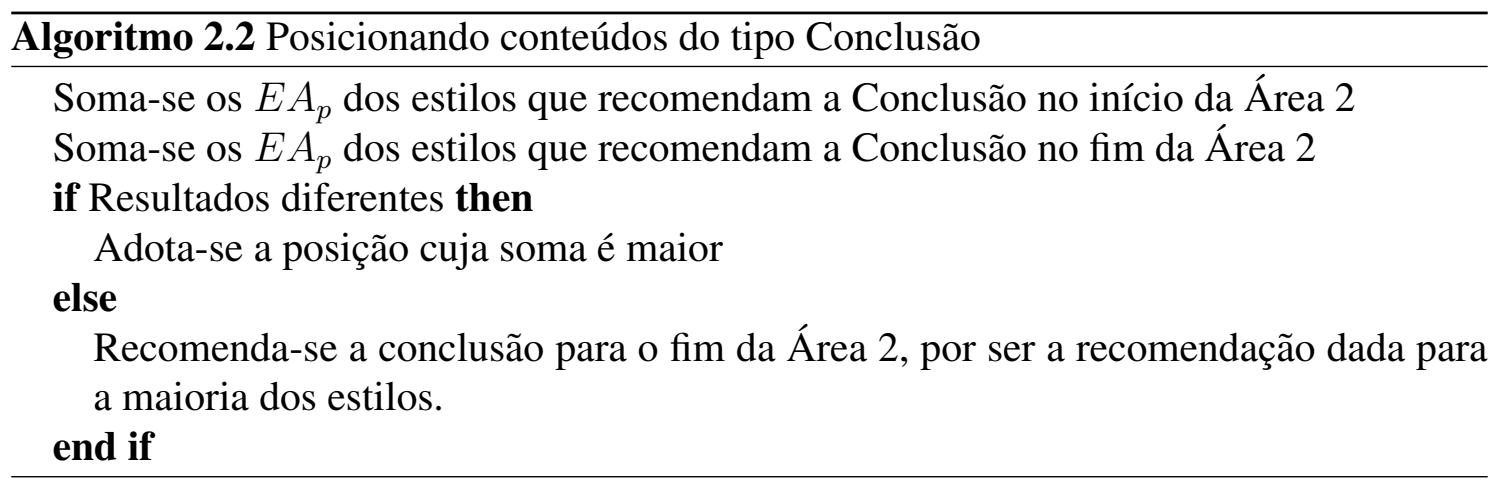

alunos), financeiros (infra-estrutura e produção de material) e tempo (um curso a distância tende a durar meses). Além disto, o teste e validação destes sistemas torna-se cada vez mais crítico, se considerarmos que a demanda por este tipo de sistemas cresce rapidamente, e o número de estudantes que dependem deles tem aumentado consideravelmente [Graf et al. 2009].

Considerando estes aspectos, apresenta-se a seguir as 9 primeiras iterações de um experimento realizado com o modelo proposto neste trabalho, considerando o modelo para simulação de desempenho do estudante apresentado em [Dorça et al. 2013b]. No ME inicial do estudante, os $E A_{p}$ foram inicializados em $50 \%$ para todos EA, em todas as dimensões, ou seja, nenhuma preferência foi inicialmente detectada. A medida que o processo evolui, o sistema atualiza as preferências do estudante, conforme abordagem apresentada em [Dorça et al. 2013a]. A cada iteração, é apresentado o resultado produzido pela abordagem proposta, assim como o valor dos $E A_{p}$ do estudante. A Figura 2 apresenta os resultados obtidos nas iterações 1 a 3 . A Figura 3 apresenta os resultados obtidos nas iterações 4 a 6 . A Figura 4 apresenta os resultados obtidos nas iterações 7 a 9.
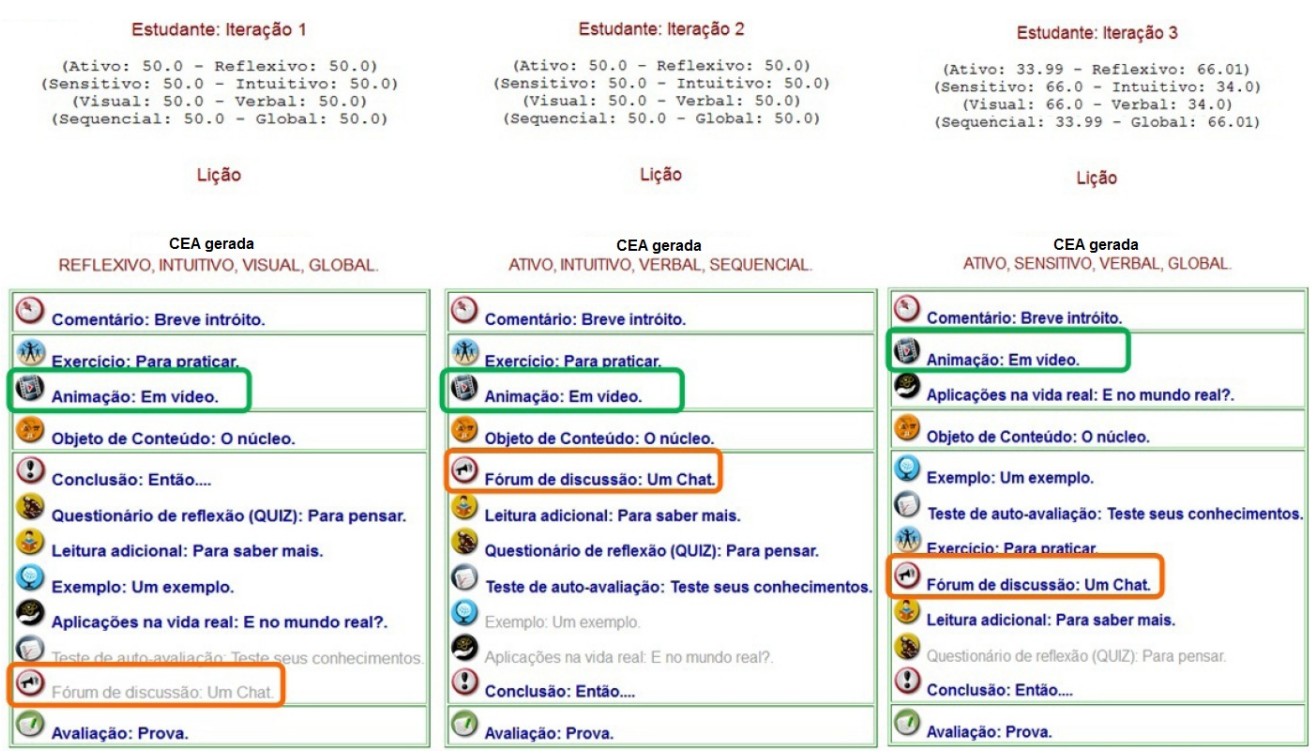

Figura 2. Adaptatividade gerada pelo modelo nas iterações 1 a 3.

Uma das características fundamentais desse processo é o comportamento estocástico. Assim, percebemos que nem sempre o EA de maior probabilidade no ME é selecionado para compor a CEA, e então, não é considerado na adaptatividade gerada. 

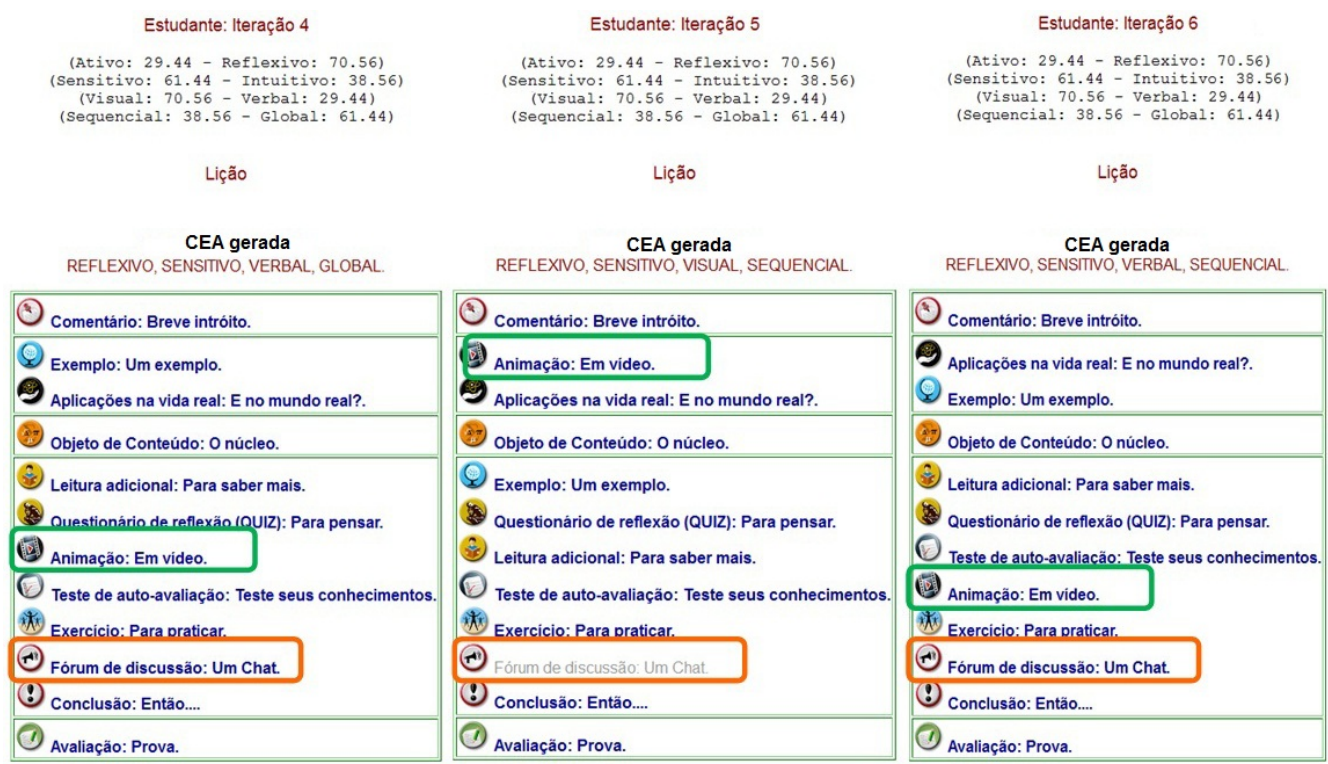

Figura 3. Adaptatividade gerada pelo modelo nas iterações 4 a 6.
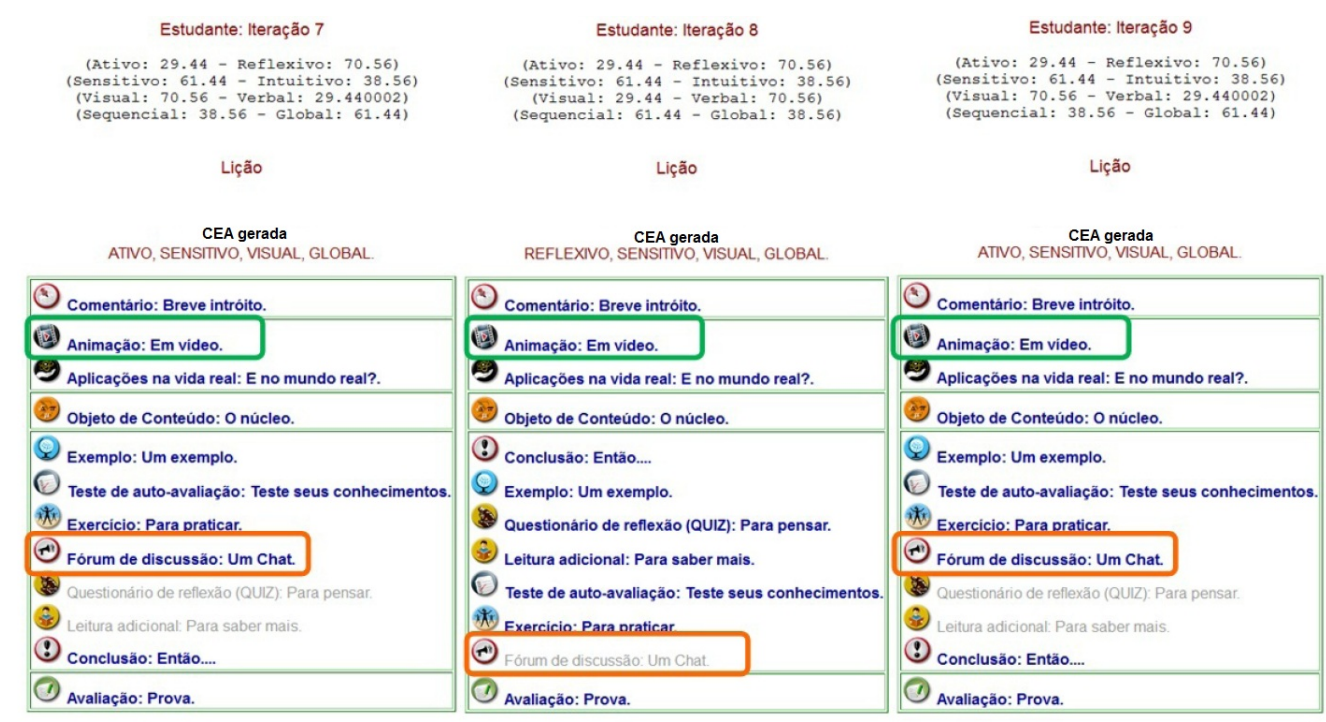

Figura 4. Adaptatividade gerada pelo modelo nas iterações 7 a 9.

Isto pode ser facilmente percebido, por exemplo, na iteração 6, Figura 3, quando foram considerados, na geração de adaptatividade, os estilos verbal e sequencial, os quais não eram, naquela iteração, os estilos de maior probabilidade em suas respectivas dimensões no ME. Vale lembrar que isso ocorre porque, na abordagem proposta, não se considera o $E A_{p}$ maior como sendo uma preferência definitiva, de forma determinística, mas sim como uma possível tendência. Isso permite que, em caso de inconsistências, as preferências corretas possam ser detectadas, tornando o processo de adaptatividade mais efetivo.

Destaca-se, ainda, ao longo das iterações apresentadas, a evolução do posicionamento de alguns objetos. O objeto Animação é recomendado como primeiro objeto da área 1. Ao longo das iterações, a posição designada para esse objeto variou, chegando a 
figurar entre os últimos conteúdos recomendados (iterações 4 e 6). Entretanto, à medida que o perfil modelado $\left(E A_{p}\right)$ converge para as preferências reais do estudante, o objeto Animação passa a ocupar sua posição adequada. Da mesma forma, o objeto Fórum de discussão, chegou a ser o primeiro objeto da área 2 (iteração 2). Ao fim das iterações, este também passou a ocupar uma posição mais adequada, considerando-se os $E A_{p}$ do estudante.

Conclui-se, então, que a abordagem apresentada neste trabalho mostrou-se completamente aderente à geração de adaptatividade para estudantes modelados através de uma abordagem automática, dinâmica e estocástica de EA. A abordagem apresentada, mesmo com um pequeno número de iterações aplicado, já demonstrou eficácia na modelagem do estudante, a adaptatividade gerada condiz com o que seria esperado para ambos os casos. Finalmente, acredita-se que os resultados obtidos por experimentos validam a abordagem proposta neste trabalho, estando então apta a ser implementada e utilizada em um ambiente existente, em um processo de ensino-aprendizagem real. A próxima seção tece algumas conclusões e aponta alguns trabalhos futuros.

\section{Conclusão e Trabalhos Futuros}

Este trabalho apresenta uma abordagem para geração automática de conteúdo personalizado através da recomendação estocástica de objetos de aprendizagem no processo de ensino em sistemas adaptativos e inteligentes para educação. Experimentos realizados mostraram resultados promissores. Os testes realizados apresentaram ordenação e destaque dos conteúdos de maneira eficiente, levando em consideração os relacionamentos estabelecidos por [Graf e Kinshuk 2010] entre os objetos e as preferências de EA dos estudantes em cada dimensão, conforme abordagem proposta por [Dorça et al. 2013a].

Tais resultados mostraram uma capacidade sólida desta abordagem em oferecer curso personalizado em ambiente de hipermídia adaptativa de forma individualizada. Um outro aspecto importante deste trabalho em relação a outras abordagens é a utilização de um ME probabilístico, atualizado constantemente através de um método baseado em aprendizagem por reforço para modelagem automática e dinâmica do estudante considerando EA, o que traz aspectos inovadores, e vários benefícios ao processo de adaptatividade, conforme atesta [Dorça et al. 2013a].

Finalmente, a abordagem proposta neste trabalho tem o foco principal no fornecimento de uma experiência de aprendizagem que atenda aos EA dos estudantes no cumprimento de objetivos de aprendizagem bem definidos de forma eficiente, o que atualmente é um desafio em pesquisas. Como trabalho futuro, tem-se a implantação deste método em um ambiente existente, tal como o Moodle (2013), para que possa ser utilizado com estudantes reais. Além disto, um objetivo é o estudo e análise de novas teorias para recomendação de conteúdos através de EA e outras características do estudante, para que se possa agregar novas regras ao modelo proposto neste trabalho, tornando o processo de adaptatividade ainda mais efetivo.

\section{Referências}

Bittencourt, I. e Costa, E. (2011). Modelos e ferramentas para a construção de sistemas educacionais adaptativos e semânticos. Revista Brasileira de Informática na Educação, 19(1):49-56. 
Brusilovsky, P., Eklund, J., e Schwarz, E. (1998). Web-based education for all: A tool for developing adaptive courseware. In Seventh International WWW Conference, pages 291-300.

Dorça, F. A., Lima, L. V., Fernandes, M. A., e Lopes, C. R. (2012). A stochastic approach for automatic and dynamic student modeling in adaptive educational systems. Informatics in Education. Vilnius University Institute of Mathematics and Informatics, Lithuanian Academy of Sciences, Lithuania. ISSN: 1648-5831., 11(2).

Dorça, F. A., Lima, L. V., Fernandes, M. A., e Lopes, C. R. (2013a). Comparing strategies for modeling students learning styles through reinforcement learning in adaptive and intelligent educational systems: An experimental analysis. Expert Systems with Applications. DOI: http://dx.doi.org/10.1016/j.eswa.2012.10.014, 40.

Dorça, F. A., Lima, L. V., Fernandes, M. A., e Lopes, C. R. (2013b). Simulação de Aprendizagem em Estudantes como Ferramenta para Minimização de Custos na Avaliação de Novas Abordagens em Sistemas Adaptativos e Inteligentes para Educação a Distância: Uma Análise Experimental. In In: XXXIII Congresso da Sociedade Brasileira de Computação (CSBC'2013). II DesafIE!. Maceió/AL.

Felder, R. e Silverman, L. (1988). Learning and teaching styles in engineering education. Journal of Engineering education, 78(7):674-681.

Graf, S. e Kinshuk, C. (2010). A Flexible Mechanism for Providing Adaptivity Based on Learning Styles in Learning Management Systems. In 10th IEEE International Conference on Advanced Learning Technologies, pages 30-34. IEEE.

Graf, S., Lan, C., Liu, T., et al. (2009). Investigations about the Effects and Effectiveness of Adaptivity for Students with Different Learning Styles. In 2009 Ninth IEEE International Conference on Advanced Learning Technologies, pages 415-419. IEEE.

Haider, M., Sinha, A., e Chaudhary, B. (2010). An Investigation of relationship between learning styles and performance of learners. International Journal of Engineering Science and Technology, 2(7):2813-2819.

Kinshuk, Liu, T., e Graf, S. (2009). Coping with Mismatched Courses: Students' behaviour and performance in courses mismatched to their learning styles. Educational Technology Research and Development, 57(6):739-752.

Nat, M., Walker, S., Bacon, L., e Dastbaz, M. (2010). Designing personalisation in LAMS. In Proceedings of the 2010 European LAMS \& Learning Design Conference.

Van Zwanenberg, N., Wilkinson, L., e Anderson, A. (2000). Felder and silverman's index of learning styles and honey and mumford's learning styles questionnaire: how do they compare and do they predict academic performance? Educational Psychology, 20(3):365-380.

Virvou, M., Manos, K., e Katsionis, G. (2003). An evaluation agent that simulates students' behaviour in intelligent tutoring systems. In IEEE International Conference on Systems, Man and Cybernetics, 2003, volume 5, pages 4872-4877. IEEE, IEEE. 